\title{
IMPORTANCIA DE LA PLANIFICACIÓN SOBRE LA RECOLECCIÓN DE LOS DATOS: APRENDIZAJES A PARTIR DE UNA EXPERIENCIA DE INVESTIGACIÓN
}

\section{IMPORTANCE OF PLANNING FOR DATA COLLECTING: LESSONS FROM A RESEARCH EXPERIENCE}

\author{
Zaida Salazar Mora* \\ Jorge Esteban Prado-Calderón ${ }^{* *}$
}

RESUMEN

\begin{abstract}
En el presente artículo se relatan las experiencias y aprendizajes logrados durante la realización de una investigación social llevada a cabo a nivel nacional. Se destaca la importancia de la planificación para las etapas posteriores consistentes en la recolección de la información y el procesamiento de los datos. Asimismo, se destaca la relevancia del trabajo coordinado con personas de otras disciplinas y se llevan a cabo algunas reflexiones sobre el sistema educativo costarricense.
\end{abstract}

PALABRAS CLAVE: RECOPILACIÓN DE DATOS * INVESTIGACIÓN DE CAMPO * MÉTODOS DE INVESTIGACIÓN * PLANIFICACIÓN * SISTEMAS DE ENSEÑANZA

\section{ABSTRACT}

In this paper we report the experiencies and learnings achieved during the enforcement of a social investigation at national level. It is emphasised the importance of planning phase for the later stages, which consist in the gathering of information and data processing. Also, we highlight the relevance of interdisciplinarity and some reflexions about the costarrican educational system.

KEYWORDS: DATA COLLECTING * FIELD RESEARCH * RESEARCH METHODS * PLANNING * EDUCATIONAL SYSTEMS

Escuela de Psicología de la Universidad de Costa Rica (UCR).

zaidasalazar@hotmail.com

** Escuela de Psicología de la Universidad de Costa Rica (UCR).

jorgepradopsi@gmail.com 


\section{INTRODUCCIÓN}

Las investigaciones científicas con el objetivo de generar conocimiento, tanto de enfoque cualitativo como cuantitativo, emplean procesos cuidadosos y sistemáticos, llevan a cabo observaciones, evalúan fenómenos, establecen suposiciones $y$ demuestran su fundamento, revisan las suposiciones sobre la base de pruebas o análisis $y$ proponen nuevas observaciones $y$ evaluaciones para fundamentar y generar nuevas ideas. Principalmente, la investigación de enfoque cuantitativo, se caracteriza por ser sistemática, controlada, ordenada, se tiene confianza crítica de los resultados y es un proceso con diversas etapas, igualmente importantes, para obtener resultados válidos y confiables, asimismo, se dice que una de las etapas fundamentales es la recolección de los datos. Esta implica elaborar un plan detallado de procedimientos para obtener la información de acuerdo a los objetivos y propósitos de la investigación (Hernández, Fernández y Baptista, 2010; Kerlinger y Lee, 2002).

En este artículo se hace especial referencia a la etapa de recolección de los datos, fase que implica determinar las fuentes de las cuales se va a obtener la información, dónde se localizan esas fuentes, definir los medios y procedimientos que se van a utilizar para obtener los datos y cómo se van a preparar para que puedan analizarse y responder con estos a las preguntas de la investigación (Hernández et ál., 2010).

Asimismo, es importante considerar los cuidados a seguir para lograr que los datos sean pertinentes $y$ de buena calidad. Los datos son la materia prima con la cual se realizará el análisis para responder a los problemas planteados en la investigación; de ellos dependen las conclusiones, las recomendaciones $y$ las decisiones que se tomen. Por consiguiente, la recolección de los datos es vital en la investigación (Gómez, 1998).

$\mathrm{El}$ investigador o investigadora debe tratar de conseguir los datos más exactos y precisos, aun sabiendo que nunca se va a conseguir información $100 \%$ objetiva. Considerando este aspecto, se debe conocer $y$ evaluar las limitaciones de la información recolectada, es decir, se debe tener una actitud crítica ante los datos.

La investigación es un proceso que requiere aplicar los pasos correspondientes y las herramientas fundamentales y por lo general, en la práctica investigativa se tiene que enfrentar a problemas específicos. El interés de este artículo es comunicar algunos aprendizajes adquiridos a partir de las condiciones que se enfrentan, propiamente en la recolección del dato, considerando la experiencia de una investigación en particular.

Entre el 4 de mayo y el 16 de agosto de 2010, se realizó el trabajo de campo de un estudio a nivel nacional, para determinar las propiedades de confiabilidad $y$ validez del Test de Actitudes hacia la Alimentación, en estudiantes de secundaria. Para ello, se aplicó el instrumento a una muestra de 4285 estudiantes de noveno, décimo y undécimo año, en 59 colegios de Costa Rica.

En el presente artículo, se describe la experiencia de dicha investigación desde la planificación del trabajo de campo hasta la digitación de los cuestionarios, con el objetivo de que nuestras experiencias, logros y errores sean de ayuda para la buena realización de futuras investigaciones.

$\mathrm{Al}$ respecto, es importante indicar que la literatura no suele abordar el tipo de recomendaciones aquí presentadas. Así, por ejemplo, libros de texto generales pero ya clásicos en nuestro contexto, como el de Hernández et ál. (2010) o el de Kerlinger y Lee (2002), describen los aspectos comunes del proceso investigativo (planteamiento, diseños, metodologías, muestreo, análisis, reportes) sin entrar en recomendaciones tan específicas sobre la recolección del dato. Asimismo, bibliografía más especializada sobre la planificación e implementación de la investigación, como los textos de BonillaCastro y Rodríguez (2005) y el de Tamayo y Tamayo (2004), mantienen una perspectiva general del proceso.

Esta situación no cambia al considerar otro tipo de publicaciones, tales como artículos, ni al abordar la literatura de los pueblos angloparlantes. Así, por ejemplo, trabajos en publicaciones periódicas sugerentemente "prácticos", como el de Abello (2009) y el de Onwuegbuzie $y$ Leech (2006), se enfocan en recomendaciones específicas para la investigación, las cuales se centran más en la elaboración de problemas y 
objetivos que en la recolección del dato. Asimismo, libros de texto utilizados en el contexto angloparlante, como el de Gray, Williamson, Karp y Dalphin (2007), aunque excelentes, se suelen enfocar en descripciones detalladas de los distintos diseños y metodologías de investigación, sin entrar en recomendaciones prácticas como las que a continuación presentamos.

\section{EXPERIENCIA DE RECOLECCIÓN DE DATOS}

\section{EL OBJETIVO DEL ESTUDIO}

La recolección del dato está vinculada al objetivo del estudio, el cual indicará la finalidad de la investigación.

Dentro de la línea de investigación de los trastornos alimenticios, como la anorexia $y$ la bulimia, se tiene clara la necesidad de contar con tests adaptados $y$ validados para realizar investigación y diagnóstico en la población joven costarricense. Es así como se propone un estudio a nivel nacional, cuyo objetivo general es validar el Test de Actitudes hacia la Alimentación (EAT-40, por sus siglas en inglés). Este instrumento fue diseñado por Garner y Garfinkel (1979) para evaluar las actitudes y conductas hacia la comida, el peso y el ejercicio, relacionadas con la anorexia nerviosa. La prueba fue posteriormente, adaptada al contexto español por Castro, Toro, Salamero y Guimerá (1991).

En Costa Rica, el EAT ha sido aplicado en algunas investigaciones sin haber obtenido evidencias de su validez y confiabilidad. Esto se considera una gran deficiencia, en tanto la investigación del comportamiento requiere una adecuada medición de las variables. La Psicometría se ha preocupado porque los tests se elaboren $y$ utilicen cumpliendo con sus características técnicas y además, considerando aspectos éticos, donde se incluyen las necesidades y derechos de las personas a quienes se les aplican los tests (Aiken, 2003).

El primer análisis del EAT en Costa Rica, se realizó como un estudio piloto (Salazar, 2008), donde se hizo un análisis cualitativo de los ítems y se aplicaron análisis de confiabilidad $y$ validez en una muestra de 174 jóvenes. Otro estudio fue en el año 2009, cuando se analizó la estructura factorial y la confiabilidad del
EAT $(n=569)$, y se examinó su efectividad para diferenciar a las mujeres con trastornos de la conducta alimentaria (Salazar, 2012). En ambas investigaciones los índices de confiabilidad $y$ validez resultaron adecuados. Sin embargo, considerando que en estos estudios las muestras son intencionales y ubicadas en el Área Metropolitana, se decide realizar un estudio a nivel nacional, cuya experiencia en la recolección de los datos se exponen a continuación.

\section{LA DEFINICIÓN DEL MARCO MUESTRAL}

Teniendo definidos claramente los objetivos de la investigación, se trabaja en la determinación del grupo de estudio. Respecto a la obtención de la muestra, un primer punto consistió en definir una población adecuada para responder al objetivo general y los objetivos específicos de la investigación. Uno de los peores errores que se puede cometer en el proceso de las investigaciones, principalmente cuantitativas, es llegar a obtener datos de sujetos $y$ de variables que no correspondan a los planteados en los objetivos, puesto que a fin de cuentas serían insumos que no van a ser útiles para los resultados que se esperan con el estudio. Esto, además de que implicaría gastos innecesarios que podrían ser invertidos en otras etapas del estudio, iría contra la ética científica, en tanto se estaría malgastando el tiempo y esfuerzo de las personas participantes.

Considerando que la muestra debe responder claramente a los objetivos de la investigación, en este proyecto se eligió como población de estudio a las personas adolescentes, habitantes del país, insertas en el sistema de educación formal, ya que una revisión exhaustiva de las investigaciones en el campo de la anorexia y bulimia, indica que la mayor prevalencia se da en esta etapa del desarrollo (Salazar, 2008).

A partir de esto, se llevó a cabo el primer contacto con profesionales externos a la investigación. Gracias a las personas encargadas del Departamento de Análisis Estadísticos del Ministerio de Educación Pública (MEP), se obtuvo un registro actualizado (año 2009) de todas las instituciones de educación secundaria del país, además de sus ubicaciones, teléfonos, 
directores $y$ cantidad de estudiantes. En toda investigación que lo amerite, es importante destacar que es imprescindible consultar los registros actualizados de las instancias correspondientes.

Con base en los detalles de estos registros, se decidió abarcar colegios públicos, privados y privados-subvencionados, así como, colegios de formación académica, técnica y artística. Por otra parte, siguiendo el objetivo de la investigación, se decidió dejar por fuera los colegios nocturnos y trabajar solo con los diurnos. La razón primordial que motiva esta decisión es la edad, ya que en los colegios nocturnos la mayoría de estudiantes son adultos jóvenes o mayores, lo cual sobrepasa la edad de la adolescencia que es el interés de la investigación.

\section{LA DEFINICIÓN DE LA MUESTRA}

En el diseño de la muestra, uno de los aspectos básicos a tomar en cuenta es la necesidad de consultar a un experto en esta área. Lo anterior considerando que las y los investigadores, por lo general, son expertos en sus campos de formación, pero no precisamente en Estadística ni específicamente, en la selección de las muestras de acuerdo a los objetivos que plantea el estudio. En este caso, la consulta al experto le da un valor de confianza al estudio, implica un aprendizaje en conjunto y los investigadores e investigadoras de ambas disciplinas (Psicología y Estadística) se enriquecen.

Para la determinación de una muestra representativa de la población de adolescentes escolarizados, se obtuvo la asesoría del M.Sc. Fernando Ramírez, estadístico especialista en selección de muestras de la Escuela de Estadística de la Universidad de Costa Rica (UCR).

A partir del padrón muestral facilitado por el MEP y con la asesoría del estadístico de la UCR, se definió un diseño aleatorio de conglomerados mediante un muestreo sistemático, usando como medida de tamaño el total de estudiantes, lo que generó una muestra de selección PPT (probabilidad proporcional al tamaño). En otras palabras, con esta técnica de muestreo, los colegios con más estudiantes tienen una mayor probabilidad de ser seleccionados en la muestra.
Para cada colegio, se decidió aplicar los instrumentos a un grupo de estudiantes de noveno año, uno de décimo año y otro de undécimo año, con el fin de encuestar a estudiantes adolescentes. De ser posible, dentro de cada colegio, estos grupos serían seleccionados al azar. Asimismo, se buscó obtener datos tanto de hombres como de mujeres, de manera que la muestra final estuvo compuesta en su mayoría por instituciones mixtas, siendo solamente una de ellas exclusiva para mujeres.

Esta muestra asumía la existencia de algunos supuestos. Primero, que en cada colegio se visitaría un grupo de cada uno de los niveles mencionados, en virtud de obtener datos de estudiantes entre 14 y 18 años de edad, según se indicaba en la información ofrecida por el MEP. Segundo, que cada grupo estaría conformado por alrededor de 25 estudiantes. Tercero, que la muestra total por colegio sería de 75 estudiantes. Por último, que todo lo anterior se asumía en tanto la matrícula del año 2010 fuera similar a la de 2009 , de la cual se contaba con la información ofrecida por el MEP.

Asimismo, se tomaron en cuenta algunos hechos conocidos para estimar la muestra. Primero, que siete de los colegios seleccionados en la muestra probabilística tenían una matrícula global inferior a 100 , por lo que en estos no se seleccionarían grupos al azar sino que se llevarían a cabo censos con todos los y las estudiantes de noveno, décimo y undécimo año de esos colegios. Segundo, que si bien se conocía que el promedio de estudiantes por grupo era de 27 , se asumiría un promedio de 25 para ser conservadores. Tercero, que previendo un margen de estudiantes que no asistan a lecciones, estudiantes que no deseen participar $y$ algunos otros posibles inconvenientes, la muestra final sería de 4200 estudiantes (estimación que, de hecho, se acercó mucho a la muestra final obtenida, de 4285 estudiantes).

Finalmente, considerando la muestra planeada y con base al muestreo probabilístico, el error máximo previsto para la estimación de proporciones sería de alrededor de $1.5 \%$, para un nivel de confianza del 95\%.

La muestra final a obtener estaría conformada por 62 colegios de Costa Rica, los cua- 
les conservarían en gran medida la distribución nacional de colegios públicos $(n=56,90.3 \%)$, privados $(n=4,6.4 \%)$ y privados-subvencionados $(n=2,3.2 \%)$ y la distribución de colegios académicos $(n=49,79.0 \%)$ y técnicos $(n=13$, $21.0 \%)$. Asimismo, se contaría con una buena representación de colegios urbanos $(n=36$, $58.1 \%)$ y rurales $(n=26,41.9 \%)$.

\section{LOS CONTACTOS Y PERMISOS}

Parte fundamental de la coordinación de algunas investigaciones es el establecimiento de contactos y la obtención de permisos con instituciones $y$ personas ajenas al proyecto. En la presente investigación, se tuvo que establecer contacto previo con todos los colegios visitados, así como obtener los permisos de sus directores $y$ directoras para ingresar a la institución e invitar a los y las estudiantes a participar en el estudio.

En un primer momento, se obtuvieron los datos de contacto oficiales utilizados por el MEP y facilitados por el Departamento de Análisis Estadísticos. Esto ayudó a avanzar en la tarea de una forma mucho más eficiente. A pesar de ello, muchos de los datos de contacto no estuvieron actualizados, por lo que hubo que obtenerlos por otros medios. En estos casos resultaron de particular utilidad las llamadas al servicio 1113, ya que aunque no tuvieran registrados los números de teléfono de muchos de los colegios, pudieron facilitarnos teléfonos de otras instituciones de la comunidad, tales como centros de salud, guardias rurales o municipalidades, en las que se logró obtener la información.

Una vez que se contó con cada dato de contacto, se procedió a realizar la llamada inicial, en la cual resultó de mucho provecho hablar directamente con la máxima autoridad del colegio (director o directora), a fin de obtener la autorización inicial y que en la institución se manejara la noción común de un compromiso conjunto con el equipo de investigación de la UCR, para llevar a cabo la aplicación de los instrumentos. En algunas ocasiones, la persona al mando delegó inmediatamente la tarea a otro funcionario o funcionaria del colegio, por lo general auxiliares administrativos o personal de orientación.
En estas llamadas fue de mucha importancia contar con un protocolo. Este comprendía la presentación de la persona que llamaba (que correspondía a asistentes de la investigación), la institución que representaba, la explicación del objetivo, la importancia de la investigación y la necesidad de utilizar grupos del colegio como parte de la muestra al azar, con el fin de obtener la información. Asimismo, se evacuaban dudas con respecto al estudio, se definían las fechas entre las cuales se llevaría a cabo la investigación y se comunicaba que la fecha $y$ hora concreta de la visita, serían informadas en las siguientes semanas, de común acuerdo.

Por lo general, se realizaban varios intentos para localizar al director o directora $y$ también en algunos casos señalaban que el permiso había que solicitarlo a la Oficina Regional del MEP, siendo este un trámite más complicado, ya que estas instancias solicitaban mayor información sobre la investigación, una reunión personal y pedían resultados de la investigación. Hay que considerar que estas llamadas implicaban hacer varios intentos para localizar a las y los directores, en diferentes horarios, aumentando el trabajo de las y los asistentes, asimismo se debe considerar el costo de las llamadas telefónicas.

Luego de establecidos los contactos iniciales $y$ verificados los permisos en las instituciones, se planificaron las fechas de las aplicaciones y las rutas que se ejecutarían. En el caso de los colegios ubicados en zonas cercanas a la capital, la planificación fue más flexible. Sin embargo, para las visitas a colegios de zonas alejadas y medio alejadas, fue imprescindible una planificación cuidadosa.

Como primer aspecto se definieron las fechas en las cuales llevar a cabo la recolección de los datos. En este punto, fue importante tomar en cuenta los calendarios de las dos instituciones involucradas (UCR y colegios), a la vez que se consideraba otra variable relevante, las condiciones del tiempo. Con base en ello, se decidió realizar las aplicaciones a partir de la tercera semana de julio, ya que en estas fechas coincidían varios aspectos positivos. Por una parte, la UCR iniciaba las vacaciones de medio periodo, por lo que se contaba con menos compromisos de parte del equipo investigador $y$ 
más recursos en la UCR, tales como el transporte. Por otra parte, en estas fechas, las y los estudiantes de secundaria se encontraban reiniciando clases luego de vacaciones de quince días, los colegios se encontraban con menos presión y era menos probable que los estudiantes no pudieran participar debido a exposiciones, exámenes, giras, etc. En síntesis, se logró encontrar fechas ideales para la aplicación de los instrumentos.

Como segundo aspecto se debieron trazar las rutas provisionales a ejecutar, de manera que se optimizara el uso del tiempo y el combustible. Estas rutas fueron elaboradas en colaboración con profesionales de la Oficina de Transportes de la UCR, quienes contribuyeron enormemente gracias a su experiencia y conocimientos en la geografía, las rutas, los plazos y el estado de las carreteras del país.

Una vez definidas las rutas fue posible contactar a las instituciones para confirmar la fecha $y$ hora de la visita. Establecer el horario implicó también trabajo extra en llamadas telefónicas, notas por correo y faxes, ya que las visitas se programaron por zonas, lo cual implicaba darle al colegio un horario fijo de visita $y$ si no podía aceptarlo, había que coordinar nuevamente con diversos colegios de la zona que estaban programados para ese mismo día.

Esta etapa implicó identificar a la persona que guiaría o se encargaría de la visita, es decir, quien recibiría a las personas de la investigación, le indicaría dónde se encontrarían los grupos y organizaría los permisos con el personal docente que tenía el grupo a cargo. En algunas instituciones, especialmente las alejadas de San José, esta persona encargada del recibimiento, también nos organizó un refrigerio, aspecto que fue sumamente gratificante para el grupo de investigación. En esta comunicación previa, se obtenía además la dirección exacta de la institución y otros posibles números de teléfono.

Finalmente, luego de definida la fecha $y$ horario de la visita, así como, el nombre de la persona encargada del recibimiento, se procedió a enviar cartas formales a los colegios en las cuales se consignaran los datos específicos de la visita. Como es de suponer, para lograr estos pasos fue necesario un trabajo previo de obtener los números de fax o los correos electrónicos de los 62 colegios y en muchos casos, los números no correspondían o no existían, o no se contaba con correo electrónico. Sin embargo, la finalización de esta tarea fue necesaria para la formalización de la visita, por lo que se trabajó hasta lograr enviar la carta formal a los colegios.

\section{EL TRANSPORTE Y HOSPEDAJE}

Para definir las rutas, se clasificaron los colegios en cercanos, medios y alejados. Los primeros, se ubican en la Gran Área Metropolitana, los segundos implicaban más de dos horas de viaje para llegar a ellos y los alejados implican tener que viajar más de cuatro horas para llegar al sitio o tener que regresar al día siguiente. Los colegios visitados pueden ser consultados en la Tabla 1. Se asistió a un total de 59 instituciones de todo el país, 26 colegios alejados, 14 colegios medios y 19 colegios cercanos. De la muestra original de 62 colegios, uno se descartó por ser nocturno (CTP nocturno de Puntarenas), con otro no fue posible establecer contacto (Liceo de Tobosi), mientras que el último no fue accesible debido a las condiciones climáticas de la zona que obstaculizaron el acceso (Liceo de Comte). 
TABLA1

LISTA DE COLEGIOS DE LA MUESTRA SEGÚN UBICACIÓN

\begin{tabular}{ll}
\hline COLEGIOS CERCANOS & \\
\hline C. Adventista & L. Moravia \\
C. Madre del Divino Pastor & L. Regional de Flores \\
C. Ricardo Fernández & L. Rodrigo Hernández \\
C. Superior de Señoritas & L. Samuel Sáenz \\
Conservatorio Castella & L. San Antonio (Coronado) \\
CTP José Figueres & L. San Antonio (Desamparados) \\
CTP San Sebastián & L. Teodoro Picado \\
L. Anastasio Alfaro & Oasis de Esperanza \\
L. Curridabat & Surí Pavas \\
L. Mauro Fernández & \\
\hline COLEGIOS MEDIOS & \\
\hline C. Ambientalista El Roble & \\
C. Palmares & L. Martha Mirambell \\
C. San Francisco & L. San Diego \\
C. San Luis Gonzaga & L. San Gabriel \\
CTP Invu Las Cañas & L. San Roque \\
L. Bolivar & L. Santa Lucía \\
L. La Virgen & L. Vicente Lachner \\
\hline COLEGIOS ALEJADOS & L. Vuelta de Jorco \\
\hline CTP Cóbano & \\
CTP Guatuso & \\
CTP La Mansión & L. Felipe Pérez \\
CTP La Suiza & L. La Alegría \\
CTP Limón & L. Liverpool \\
CTP Pococí & L. Matina \\
CTP Ricardo Castro & L. Nuestra Sra. de Los Ángeles \\
CTP Sabalito & L. Nueva Guatemala \\
CTP San Carlos & L. San Ambrosio \\
CTP San Isidro & L. San Carlos \\
L. Aguas Claras & L. San Pedro \\
L. Cariari & L. Santa Cruz \\
L. Emiliano Odio & L. Santa Teresita \\
\hline & L. Sinaí \\
\hline & L. Yolanda Oreamuno \\
\hline
\end{tabular}

Fuente: Elaboración propia.

Como se explicó anteriormente, el transporte para visitar los 59 colegios fue organizado en conjunto con la Oficina de Transportes de la UCR. Nuevamente en esta etapa se considera importante la consulta de expertos en este campo que conocen las rutas y los tiempos. Las rutas se organizaron de tal manera que se visitaron uno o dos colegios en ubicación media y se seguía a uno de ubicación alejada, en este sitio se pasaba la noche para al día siguiente visitar un colegio de ubicación alejada y de allí venir hacia otros de ubicación catalogada como media y cercana.
Así, por ejemplo, un lunes partíamos a las 4:00 a.m. para iniciar aplicando cuestionarios en un colegio de Nicoya a las 8:00 a.m., seguir con colegios de Santa Cruz y Liberia; se dormía en Liberia y al día siguiente de regreso, pasábamos a colegios de Esparza, Orotina, Atenas y Palmares. Por su parte, un miércoles partíamos a las 5:00 a.m. para iniciar en un colegio de Turrialba a las 7:00 a.m., seguir con colegios de Siquirres, Matina y Limón; dormir en Limón y al siguiente día de regreso, aplicar cuestionarios en colegios de Limón, Guápiles y Pococí. 
Para el hospedaje también se recibió la recomendación de la Oficina de Transportes y de las averiguaciones que el mismo chofer hacía en las zonas de trabajo, mientras el equipo de investigadores recogía la información.

\section{LA IMPLEMENTACIÓN: RECOLECCIÓN DE LOS DATOS}

Luego de toda la fase de planificación, se procedía a la implementación, es decir, la recolección de los datos. En esta fase se llevaron a cabo muchos aprendizajes; bastantes de estos ayudaron a confirmar la importancia de las etapas previas de planificación. Asimismo, otros hicieron comprender que la planificación no cesa y que incluso al momento de la implementación, es necesario llevar a cabo acciones para adaptarse a las nuevas circunstancias.

Acorde con lo presentado anteriormente, se evidenció la importancia de tomar en cuenta la época en que se aplicarían los instrumentos. Además de calcular previamente aspectos tales como: calendario escolar, días feriados, vacaciones, estado del tiempo, cierre de carreteras, etc., en algunas ocasiones se encontraron situaciones imprevistas tales como: huelgas, retiros espirituales, elecciones estudiantiles, actos cívi$\cos y$ actividades culturales.

En muchas de estas situaciones, el mantener una comunicación constante con las personas encargadas en los colegios, previo a la visita, permitió adaptarse sin contratiempo. Sin embargo, también hubo ocasiones en que las situaciones imprevistas conllevaron atrasos inminentes: en algunos pocos casos no aparecían las personas de contacto y había que dar largas explicaciones de lo que se estaba haciendo en el colegio, o había que esperar que los grupos se desocuparan de una actividad o terminaran una clase para poder trabajar con ellos. En todas estas situaciones fue muy importante portar copias de las cartas enviadas y el nombre y puesto de las personas contacto para aportar evidencia de la confirmación de la visita. En estos contratiempos se podía tardar hasta casi dos horas en un colegio o en menos ocasiones, tener que volver otro día a trabajar con los grupos de esa institución. No obstante, en este sentido, el hecho de que estas situaciones fueran la excepción demuestra el importante papel de la planificación y la comunicación constante.

Por otra parte, se destaca la importancia de contar con un buen equipo humano y con funciones acordadas previamente, pero también flexibles $y$ adaptables a las circunstancias. En la presente investigación, un papel determinante lo desempeñó el chofer, ya que por lo general, las distancias fueron largas $y$ hubiera sido sumamente cansado para un investigador o investigadora, después de manejar cierto número de horas (cuatro o más), trabajar y continuar manejando por más tiempo. Además, la presencia del chofer ayudó a ubicar los lugares con mayor facilidad, gracias a lo cual no se perdió tiempo cuando hubo que visitar varios sitios en un mismo día.

Asimismo, en esta investigación fue muy importante la presencia de varias personas para recolectar los datos. El equipo estaba compuesto por el chofer, dos asistentes $y$ la investigadora principal. Esto implicaba que se pudieran atender tres grupos de estudio a la vez $y$ no se durara tanto tiempo en cada colegio. $\mathrm{Si}$ se lograba trabajar con tres grupos al mismo tiempo, la duración estaba cercana a una hora. En esta tarea en específico, la utilización de teléfonos celulares fue de gran utilidad, pues permitía coordinar entre la investigadora principal, los asistentes y el chofer en cualquier momento del proceso.

Otro aspecto fundamental fue que cada miembro del equipo tuviera un rol determinado, tal como ubicar la próxima ubicación, averiguar el tiempo de duración para llegar a esta, ubicar un sitio para comer $y$ dormir (chofer); establecer el contacto con la dirección del colegio y dar los agradecimientos, entregar a la dirección una copia del consentimiento informado $y$ datos de contacto del equipo investigador; llevar el control de los instrumentos completados, según colegio y sexo, en este caso, para controlar la muestra, tomar nota de otros aspectos de la zona, el colegio y de nuevos contactos en este, etc. En este caso, las funciones fueron moldeadas desde las primeras aplicaciones, lo que permitió una implementación mucho más ágil a la hora de iniciar con estas en los colegios alejados $y$ medios. 
En otro orden, se considera recomendable en estas giras por el país, llevar meriendas por los atrasos que se dan y por las distancias para conseguir sitios donde comer. Asimismo, llevar ropa para diversos estados del tiempo, ya que se dan cambios abruptos de calor a frío y de lluvias. Por consiguiente, es conveniente también llevar medicinas.

En el mismo sentido, fue imprescindible llevar preparados todos los materiales de recolección. En esta investigación, estos materiales estuvieron conformados por los cuestionarios, las fórmulas de consentimiento informado $y$ otros documentos como tablas de seguimiento y cronogramas. En muchas de las comunidades a las que se asistió no se contaba con los recursos para, por ejemplo, fotocopiar cuestionarios o imprimir nuevas fórmulas de consentimiento informado, por lo que contar con todos los materiales desde el inicio de la jornada fue sumamente útil.

En general, la aplicación de los instrumentos se llevó a cabo de una manera eficiente, como se explicó anteriormente, logrando completar la muestra deseada. Sin embargo, vale señalar que se estaba trabajando con una población adolescente escolarizada y uno de los factores que afectó la recolección de los datos en algunos pocos colegios, fue la indisciplina $y$ falta de autoridad del docente para con sus estudiantes. Asimismo, otros inconvenientes fueron las llegadas tardías de estudiantes y el uso del teléfono celular. Esto implicó que el investigador o investigadora tuviera que establecer un nuevo orden en la clase, para obtener respeto $y$ lograr la atención del grupo, por ejemplo, dar explicaciones más detalladas, establecer empatía con el grupo, aclarar que no se debe usar el celular mientras se está en la aplicación de la prueba, entre otros. Se eliminaron varios cuestionarios de estudiantes que se evidenciaba que no estaban concentrados en la tarea, a pesar de aprobar el consentimiento informado. Esta conducta se observó principalmente en los colegios públicos.

En el otro extremo, también se encontró con casos de estudiantes deseosos de colaborar a pesar de dificultades, tales como, adecuaciones curriculares o estudiantes extranjeros que comprendían muy poco del idioma español. En estos casos se apoyó a los y las estudiantes para que completaran el cuestionario, pero sus datos no se tomaron en cuenta en la muestra debido a que se evidenció una gran dificultad para comprender los ítems.

Finalmente, en un diseño ideal de muestreo para investigaciones como esta, los colegios son seleccionados aleatoriamente, las secciones de cada nivel del colegio también son seleccionadas al azar e incluso, los y las participantes dentro de cada aula pueden ser seleccionados de manera aleatoria. En esta investigación, los colegios elegidos efectivamente fueron seleccionados al azar (como se explicó en un subapartado anterior); sin embargo, las secciones no pudieron ser escogidas de esta forma. Se seleccionaron los grupos que estaban presentes el día de la visita y que estaban disponibles en ese momento, por ejemplo, los que estaban iniciando la clase para tener mayor tiempo con el grupo o en ocasiones, los que no estaban en examen; es decir, donde se dieran las mejores condiciones para aplicar los instrumentos. Posteriormente, el cuestionario se aplicó a todos los $y$ las estudiantes que conformaban cada grupo (aunque en los análisis estadísticos posteriores se podrán llevar a cabo repeticiones de los análisis con submuestras al azar para corroborar la consistencia de los resultados).

\section{LA DIGITACIÓN DE LOS DATOS}

En el caso de las investigaciones con enfoque cuantitativo, como la presente, una actividad en la que la planificación es imprescindible, corresponde a la etapa de sistematización de la información. Dentro de esta, la introducción de los datos en un paquete estadístico (por ejemplo, el SPSS) también puede ser planificada de manera que se optimice el procedimiento.

Esta etapa de planificación es aun más relevante cuando se manejan grandes cantidades de información. Por ejemplo, en la investigación que se llevó a cabo, se obtuvo un total de 4285 cuestionarios, cada uno con 186 reactivos, para un total de 797010 valores. Durante la fase de digitación de estos cuestionarios, se realizaron varios aprendizajes, los cuales se 
consideran útiles para futuras investigaciones $y$ que son poco tratados en la literatura.

Primero que nada, es aconsejable contar con el hardware adecuado, utilizar teclado numérico y que el equipo cuente con un procesador lo suficientemente veloz para no entorpecer el trabajo. Asimismo, un elemento importante es obtener el software adecuado y poder ajustarlo a las necesidades de digitación. Por ejemplo, en el presente estudio se observó que para cada nuevo caso, el sPSS no registraba adecuadamente uno de los valores digitados cerca del ítem 90, aunque el digitador lo hubiera introducido correctamente. Esto ocasionaba que el digitador se tuviera que devolver a introducir nuevamente el valor por cada nuevo cuestionario, lo cual quitaba un par de segundos en cada caso, pero hubiera requerido varias horas al considerar el trabajo total. El problema se solucionó instalando una versión más reciente del programa, en la cual no se daba este inconveniente.

Por otra parte, al ser una encuesta de gran tamaño, nos resultó conveniente evitar en la medida de lo posible, las variables de cadena (texto) y precodificar todas como variables numéricas, aunque sus valores fueran nominales (por ejemplo, provincias, nombres de colegios, etc.). Esto permitió ahorrar una cantidad de tiempo muy considerable $y$ reducir enormemente la probabilidad de errores en la digitación. Asimismo, evidenciamos la conveniencia de preferir las teclas más cómodas del teclado numérico en la precodificación $(0,1,2,3)$, aun sobre los valores reales que luego, adoptaran en la posterior codificación de los instrumentos.

Finalmente, en esta investigación fue de utilidad calcular el tiempo que tardaría la digitación de toda la información, a fin de determinar si se lograría cumplir con los plazos. Así, a partir de la digitación de los primeros 200 cuestionarios se logró determinar el promedio de tiempo por caso y de esta manera calcular una proyección del tiempo necesario para digitar el total de cuestionarios. Con base en ello, se logró determinar que se necesitarían más digitadores, por lo que se contó con cuatro asistentes, uno de los cuales fungió como supervisor del trabajo de los otros, depurando la base de datos y evaluando la fiabilidad de la digitación por medio de muestreos de casos al azar.

En esta etapa se abarcaron otras tareas previas como la selección de las y los digitadores, considerando aspectos como el manejo del programa de cómputo, la concentración, la responsabilidad, la disponibilidad del tiempo $y$ horario de trabajo, así como también contar con un supervisor de digitadores. Todo esto implica recursos que es necesario tener calculados en el presupuesto de la investigación. En el caso de este estudio, no se tenía claro el tiempo ni el costo de esta etapa, por lo cual fue necesario conseguir presupuesto extra para poder cumplir con la misma, tarea que se vuelve difícil y puede atrasar considerablemente la investigación.

\section{REFLEXIONES SOBRE EL SISTEMA EDUCATIVO NACIONAL}

Más allá de los aprendizajes sobre el proceso de la investigación y la recolección de los datos, el visitar varios colegios (59 instituciones) en diferentes zonas del país, siendo colegios públicos y privados, permitió reflexionar sobre el sistema educativo nacional. Preliminarmente al análisis estadístico que se va a realizar sobre los datos de esta investigación $y$ de acuerdo a la observación del equipo que recolecta los datos, se puede decir que hay un significativo contraste en variables como el tipo de colegio, ya sea público o privado, las zonas de ubicación, así como en el ejercicio de la educación por parte de docentes, directores $y$ directoras.

Es así como, haciendo un análisis cualitativo de las situaciones de estos colegios, se observó diferencias significativas entre los recursos de los colegios privados y los públicos. Los primeros gozan de mejores condiciones materiales, por ejemplo, aire acondicionado en las aulas, ventanas con protección de insectos, equipo de computadora y video beam en cada aula, mayores espacios para la recreación, mejores condiciones en el mobiliario, paredes pintadas, piso de mosaico, entre otras. Por su parte, se observa un enorme contraste en las instituciones públicas cuando se encontró con aulas cuyo piso y paredes son de concreto 
expuesto, con muy poca ventilación y luz, sin pupitres (los estudiantes para sentarse tienen que buscar pupitres en otras aulas, que por lo general están en mal estado); son espacios que dan la impresión de ser bodegas. Se da el caso específico de un colegio con enormes huecos en sus paredes o aquel que funciona en un "galerón" que corresponde a un centro comunal y en el cual funcionan todos los niveles (de primero a quinto), cada uno en una esquina del mismo, sin paredes que dividan los espacios.

Las condiciones materiales de las instituciones también se diferenciaron ampliamente según su ubicación geográfica. Los colegios ubicados en la Región Central y en zonas urbanas, tenían mejores condiciones físicas que los ubicados en zonas rurales y periféricas. Asimismo, dentro de las zonas fuera del Gran Área Metropolitana, los colegios ubicados en la zona noreste (Huetar Atlántica) y sureste (Brunca) del país, presentaron condiciones de infraestructura inferiores a las de otras zonas periféricas. Esto concuerda con la información socioeconómica ofrecida por documentos como el Decimoséptimo Informe Estado de la Nación en Desarrollo Humano Sostenible (Programa Estado de la Nación, 2011) y la Encuesta Nacional de Hogares 2011 (Instituto Nacional de Estadística y Censos, 2012). Estas publicaciones afirman consistentemente que la mayor incidencia de la pobre$z a$, pobreza extrema y desempleo abierto se viene dando en las zonas periféricas (Brunca, Chorotega y Pacífico Central), asimismo, es en estas zonas donde se dan las peores condiciones de hacinamiento o deterioro en las viviendas (Huetar Atlántica), pérdida de puestos de trabajo (Chorotega) y semiinformalidad laboral (Huetar Atlántica y Chorotega).

De igual manera, en los mismos colegios se encuentran grandes contrastes; por ejemplo, en el grupo de docentes se encontró aquellos que hemos llamado modelos, que realizan grandes esfuerzos personales para lograr mejores procesos de enseñanza; entonces, por ejemplo, llevan su propio equipo de computadora y video beam a la clase todos los días, esto viajando en bicicleta, recorriendo kilómetros, cruzando puentes peligrosos y lugares donde hay riesgo de ser asaltado, para poder ofrecer lecciones más dinámicas e interesantes a sus estudiantes. Otro ejemplo de profesor modelo fue un docente de matemáticas que da clase en verano a sus estudiantes, cobrando una módica suma de dinero para dotar el aula con las condiciones necesarias. Contrariamente, se observan también otros docentes que no les importa la suciedad del aula, la indisciplina ni en general, sus estudiantes.

En relación con el liderazgo y manejo de la dirección, se observan colegios públicos que parecen contar con mejores recursos $y$ hay en ellos una buena organización de la gestión; es decir, la persona en la dirección es muy organizada y realiza grandes esfuerzos para conseguir recursos, mantener a la población de docentes y estudiantes identificados y motivados por desarrollar la institución a la que pertenecen. De igual manera, hay colegios con una estructura deficiente, presentando en ellos problemas de organización y coordinación. Pareciera ser que las direcciones verdaderamente juegan un papel muy importante de liderazgo en el desarrollo de la institución.

Por otra parte, las y los estudiantes en su mayoría, tuvieron actitudes colaboradoras con la investigación; sin embargo, se observa un mejor manejo de la disciplina en colegios privados que en públicos, por ejemplo, en los privados no se permite el uso de celular en la clase $y$ en contraste se encuentran colegios públicos donde el docente informa que no le puede retirar el teléfono al estudiante durante la clase porque puede recibir una demanda por parte de este. De igual manera, en los colegios públicos no hay una reglamentación de uso del celular en el aula.

Finalmente, algunos docentes de los colegios públicos se quejan y comparten con los investigadores su criterio sobre el estado de la educación secundaria costarricense, haciendo críticas concretas al sistema de "arrastre" de materias, el sistema de becas estudiantiles $y$ el poco o nulo apoyo que reciben por parte del Estado para dotar de recursos a estas instituciones públicas, entre muchas otras. En general, nos quedó la percepción de que las y los docentes tienen suficiente conocimiento $y$ 
experiencia sobre su quehacer, no obstante, sus criterios no son tomados en cuenta por el MEP a la hora de dictar políticas y hacer cambios. Además, no se consideran las condiciones de las diferentes poblaciones en las medidas que se toman; por ejemplo, es muy diferente la situación de un estudiante que vive en una zona urbana cuyo colegio está cercano a su lugar de habitación, en contraste con la situación de aquellos que tienen que caminar kilómetros para llegar al mismo.

\section{CONCLUSIONES}

La experiencia de esta investigación aporta variedad de enseñanzas que van desde aspectos científicos del manejo de las muestras $y$ los datos, hasta la reflexión sobre el estado de la educación secundaria costarricense. Pensamos que este debe ser el papel del investigador o investigadora en el sentido de contextualizar su estudio y ver más allá propiamente de los datos recolectados con un fin determinado. En este caso, el gran objetivo del estudio es evaluar la validez y confiabilidad de un test para estimar el riesgo de trastornos alimenticios en población adolescente, pero nos encontramos con un contexto educativo que nos hace pensar más allá de nuestro objetivo. Dichosamente, en esta investigación se midieron también otras variables de tipo socioeconómicas, de salud y psicológicas, que van a permitir presentar datos sobre el estado general de la población adolescente costarricense; sin embargo, en esta etapa de la investigación se pueden presentar observaciones importantes que constituyen hipótesis para futuros estudios.

Como parte del aprendizaje en esta investigación, se encuentra la importancia de manejar cuidadosamente cada uno de los pasos de la investigación y particularmente, la etapa de la recolección del dato. Este manejo va a redundar en validez y confiabilidad del estudio, así como, en la buena administración del tiempo $y$ los recursos, aspectos sumamente valorados en la administración de la investigación, que en algunos casos las dejan inconclusas, perdiendo recursos $y$ hasta faltando con aspectos éticos, o durando muchos años para poder terminarlas y publicar sus resultados.
Se aprende también que por más sencilla que sea una investigación, por lo general, se requiere coordinar con otras profesiones; es decir, en mayor o menor grado trabajar interdisciplinariamente. En este caso, como profesionales en Psicología, fue necesario contar con el apoyo de educadores, estadísticos, choferes, directores, orientadores, auxiliares y demás administrativos. Esto se convierte en una riqueza para la investigación y educa o moldea al investigador o investigadora en sus actitudes. Propiamente en este estudio, al ser una población adolescente, al recoger el dato en centros educativos, al tener que trasladarse a zonas retiradas del país, entre otros aspectos, implicó ciertas conductas y actitudes del equipo de trabajo, tales como, tener paciencia, ya que hay que esperar, llamar muchas veces, insistir, pedir, explicar y ser perseverante; asimismo, tener resistencia física, madrugar, trabajar durante largas jornadas, trabajar bajo diferentes condiciones climáticas, etc.; tener que ser ordenados, sistemáticos y disciplinados; también, el equipo de investigación tiene que apoyarse y ayudarse. Todo esto cuando se tiene la meta de obtener la información en un tiempo determinado y cuando no se cuenta con gran cantidad de recursos para contratar más equipos de trabajo. Vale señalar que en Costa Rica, los recursos para la investigación no están fácilmente disponibles, por consiguiente, cuando se quiere hacer estudios con muestras más grandes y al azar, se requiere un mayor control de los recursos y mayor esfuerzo del equipo investigador.

Como se pudo observar, la muestra de esta investigación abarca población nacional, por consiguiente, es amplia y la recolección del dato se hace más laboriosa. Sin embargo, es un proceso que debe establecerse siguiendo los procedimientos científicos y controlando la mayor cantidad de variables intervinientes, ya que se trata de una prueba psicológica que se usa para tomar decisiones con respecto a la salud de las personas y se debe contar con la seguridad de que el instrumento reúna dos requisitos esenciales de la Psicometría, a saber: la confiabilidad $y$ la validez. 
IV. REFERENCIAS BIBLIOGRÁFICAS

A) LIBROS

Aiken, Lewis (1996). Test psicológicos y evaluación. 11ra. edición. México: Pearson Educación, 2003.

Bonilla-Castro, Elssy y Rodríguez, Penélope (1995). Más allá del dilema de los métodos: la investigación en ciencias sociales. 3ra. edición. Bogotá, Colombia: Norma, 2005.

Gómez, Miguel (1979). Elementos de estadística descriptiva. 3ra. edición. San José: EUNED, 1998.

Gray, Paul S.; Williamson, John B.; Karp, David A. y Dalphin, John R. There search imagination: an introduction to qualitative and quantitative methods. Cambridge, Reino Unido: Cambridge University Press, 2007.

Hernández, Roberto; Fernández, Carlos y Baptista, María del Pilar (1991). Metodología de la investigación. 5ta. edición. México: McGraw-Hill, 2010.

Kerlinger, Fred y Lee, Howard (1964). Investigación del comportamiento. 4ta. edición. México: McGraw-Hill, 2002.

Tamayo y Tamayo, Mario (2001). El proceso de la investigación científica: incluye evaluación y administración de proyectos de investigación. 4a. edición. México DF, México: Limusa, 2004.

\section{B) REVISTAS}

Abello, Raimundo. "La investigación en ciencias sociales: sugerencias prácticas sobre el proceso". Investigación y Desarrollo 17 (1). Barranquilla, Colombia. Centro de Investigaciones en Desarrollo Humano de la Universidad del Norte, 2009: 208229.

Castro, Josefina; Toro, Josep; Salamero, Manel y Guimerá, Elena. "The eating attitudes test: validation of the spanish version". Psychological Assessment 7 (2). Subdivisión de Psiquiatría del Hospital Clínico y Provincial de Barcelona, 1991: 175-190.

Garner, David y Garfinkel, Paul. "The eating attitudes test: an index of the symptomas of anorexia nervosa". Psychological Medicine 9 (22). Department of Psychiatry, University of Toronto. 1979: 273-279.

Onwuegbuzie, Anthony J. y Leech, Nancy L. "Linking research questions to mixed methods data analysis procedures". The Qualitative Report 11 (3). University of South Florida, 2006: 474-498.

Salazar, Zaida. "La escala de actitudes hacia la alimentación en Costa Rica: primeras evidencias de validez y confiabilidad". Actualidades en Psicología 26 (113). Universidad de Costa Rica, 2012: 51-71.

C) TESIS

Salazar, Zaida. "Modelos televisivos y su relación con los trastornos de la conducta alimentaria, en adolescentes de ambos sexos del área metropolitana de San José". [Tesis de Maestría en Comunicación]. Universidad de Costa Rica, 2008.

D) OTROS

Programa Estado de la Nación. Decimoséptimo Informe Estado de la Nación en Desarrollo Humano Sostenible 2010. 2011. En: <http:// www.estadonacion. or.cr>.

Instituto Nacional de Estadística y Censos. Descripción de los principales resultados de la Encuesta Nacional de Hogares 2011. 2012. En: <http://www.inec.go.cr>.

Fecha de ingreso: 20/07/2012 Fecha de aprobación: 14/01/2013 
